\title{
Anabases
}

ANABASES Traditions et réceptions de l'Antiquité

$25 \mid 2017$

Varia

\section{Curiosa (1)}

Claude Aziza

\section{(2) OpenEdition}

Journals

Édition électronique

URL : http://journals.openedition.org/anabases/6104

DOI : 10.4000/anabases.6104

ISSN : 2256-9421

\section{Éditeur}

E.R.A.S.M.E.

\section{Édition imprimée}

Date de publication : 1 avril 2017

Pagination : 163-164

ISSN : 1774-4296

\section{Référence électronique}

Claude Aziza, «Curiosa (1)», Anabases [En ligne], 25 | 2017, mis en ligne le 01 avril 2020, consulté le 20 janvier 2021. URL : http://journals.openedition.org/anabases/6104; DOI : https://doi.org/10.4000/ anabases.6104 


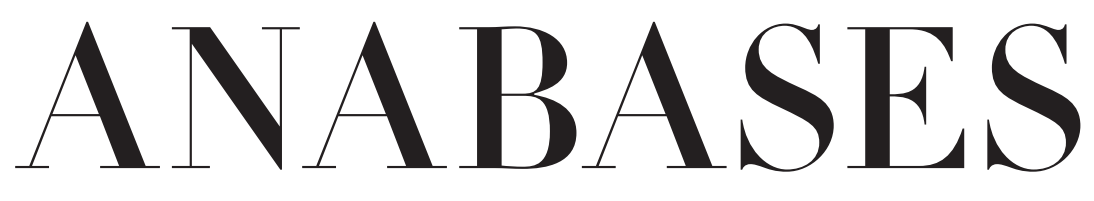

Traditions et Réceptions de l'Antiquité

$$
\begin{aligned}
& N \circ 25 \\
& 2017
\end{aligned}
$$

\section{E.R.A.S.M.E.}

Université Toulouse - Jean Jaurès 



\section{Historiographie et identités culturelles}

Étienne MaIgnan

Un lycéen pas comme les autres à l'école des Anciens :

le « Parallèle littéraire » inédit entre les Iphigénie d’Euripide

et de Racine par Charles Maurras . . . . . . . . . . . . . . . II

Aude Cohen-Skalli

Walter Spoerri (I927-20i6) et les études sur Diodore de Sicile . . . . . . . 59

\section{Traditions du patrimoine antique}

Michel LaCAVE

Anabases en France igoo-20I5. Pour une étude de la réception

de l'Anabase de Xénophon en France : doctrines,

opérations militaires et stratégies . . . . . . . . . . 7I

Loïc Marcou

La réception de l'Antiquité grecque dans le roman policier

néo-hellénique, de Yannis Maris à Petros Markaris . . . . . . . . . . . 95

\section{Archéologie des savoirs}

Romain Millot

Catilina pour combien de temps encore?

Actualités bibliographiques sur un conspirateur trop connu. . . . . . . . III

Thibaud LANFranchi

La République romaine était-elle une république ?. . . . . . . . . . . . I37 


\section{Actualités et débats}

Claude Aziza

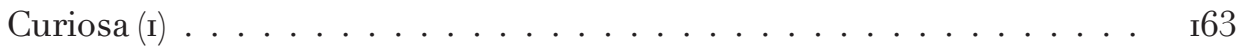

Christine Van Cauwenberghe-HoËT

Marguerite Yourcenar et l'empereur Hadrien,

une réécriture de l'histoire. Exposition au Forum antique de Bavay,

musée archéologique du Département du Nord,

du 4 février au 30 août 20r6. . . . . . . . . . . . . . . . . I 65

Claude AzızA

Antiquités parallèles (6) - Un Jésus insolite. . . . . . . . . . . . . I7 I

\section{Relire les classiques des sciences de l'Antiquité}

Giorgio FerRI

Perché leggere ancora « Quirinus. Una divinità romana

alla luce della comparazione storica » di Angelo Brelich ? . . . . . . . . .

Angelo Brelich

Quirinus. " Una divinità romana alla luce della comparazione storica »,

Studi e Materiali di Storia delle Religioni» XXXI, ı66o, p. 63-ıı . . . . . .

\section{L'atelier de I'histoire : chantiers historiographiques}

L'Antiquité au musée (coordonné par Adeline Grand-Clément) (8)

Pascal CAPus

Chiragan : une nouvelle présentation

des œuvres au Musée Saint-Raymond.

Antiquité et fictions contemporaines (coordonné par Oliviers Devillers) (12)

Silvia STUCCHI

L'image de Rome et de l'empire dans les romans

de Danila Comastri Montanari . . . . . . . . . . . . . . . . . . . . 258

\section{Comptes rendus}

Malika Bastin-Hammou et Charalampos Orfanos (dir.),

Carnaval et comédie (A. Ballabriga) . . . . . . . . . . . 273

Sophie BAsch (éd.), Portraits de Victor Bérard :

actes du colloque international organisé

à l'École française d'Athènes (5-6 avril 2013) (A. Fenet) . . . . . . . . . . . 275 
David J. Breeze, The Roman Army (B. Rossignol) . . . . . . . . . . . . . . . . 277

Luciano CANFora, Il presente come storia. Perché il passato ci chiarische le idee (M. Suzuki) . . . . . . . . . . . . . 278

Juan Ramón carbo Garcia, Apropiaciones de la Antigüedad. De getas, godos, Reyes Católicos, yugos yflechas (G. Reimond) . . . . . . . 28I

Franz Cumont, Astrologie (A. C. D. Panaino) . . . . . . . . . . . . . . . . . 282

Koen De Temmerman et Kristoffel Demoen (éd.), Writing biography in Greece and Rome: narrative technique andfictionalization (M. Cambron-Goulet) .. . . . . . . . . . . . . . . . . . 284

Hans-Christian GüNTHER (dir.), Augustus und Rom : 2000 Jahre Danach (C. Landrea) . . . . . . . . . . . . 286

Steven Hunt, Starting to Teach Latin (Cl. Aziza) . . . . . . . . . . . . . . . . . 287

Ana Iriarte y Luísa De Nazare Ferreiera (coords.), Idades e género na literatura e na arte da Grécia antiga (B. Méndez Santiago) . . . . . . . 288

M. Jankowiak et F. Montinaro, Studies in Theophanes (O. Gengler) . . . . . . 290

Barbara Levick, Catiline (C. Landrea) . . . . . . . . . . . . . . . . . . . . 292

Bruce Lincoln, Politique du paradis. Religion et empire en Perse achéménide (C. Bonnet) . . . . . . . . . . . . . . . . . . . . 294

C. Pisano, Hermes, lo scettro, l'ariete. Configurazioni mitiche della regalità nella Grecia antica (D. Bonanno) . . . . . . . . . . . . . . . 295

Procope de Césaree, Histoire des Goths (O. Gengler) . . . . . . . . . . . . . . 297

Stéphane Ratti, $L$ 'Histoire Auguste. Les païens et les chrétiens dans l'Antiquité tardive (R. Loriol) . . . . . . . . 298

Federico Santangelo, Marius (C. Landrea) . . . . . . . . . . . . . . 3oi

Marisa Tortorelli Ghidini (a cura di), Aurum. Funzioni e simbologie dell'oro nelle culture del Mediterraneo antico (C. Bonnet) . . . . . . . . . . 302

Thijs Weststeisn, Art and Antiquity in the Netherlands and Britain. The Vernacular Arcadia of Franciscus Junius (I59I-I677) (P. Butti de Lima) 3o3 Résumés. . . . . . . . . . . . . . . . . . . . . 305 Index ................................ 3II Errata. . . . . . . . . . . . . . . . . . . . 315 



\section{Actualités et débats}





\section{Curiosa (1)}

Claude Aziza

Il y avait au хvII siècle, chez des particuliers curieux de sciences et de techniques, ce qu'on nommait des « cabinets de curiosité». Chez d'autres, qu'on appelait alors des "antiquaires», des objets antiques provenant souvant d'ailleurs de pillages archéologiques. Pus modestement, au xxe siècle on trouvait dans bien des quotidiens une rubrique qui avait pour nom : "le coin des curieux». C'est au carrefour de cette triade qui n'est pas capitoline que se voudrait cette modeste rubrique à laquelle j'ai donné le nom de "Curiosa». On y trouvera, sous une forme brève, des anecdotes, des analyses, des récits sur les productions fictionnelles autour de l'Antiquité, le plus souvent cinématographiques. Ici, un sottisier, là, un film étrange, ailleurs, une thématique amusante. Telle quelle, cette mini-rubrique devrait faire sourire, ce qui par les temps qui courent n'est pas négligeable. Je serais ravi d'ailleurs si un lien s'établissait ainsi avec les lecteurs d'Anabases qui pourraient me poser les questions pour satisfaire leurs curiosités. Rêvons...

\section{Chantons d'abord sur l'air de " La première fois "...}

Le chariot-travelling fut utilisé pour la première fois dans Cabiria (Giovanni Pastrone, 1912-1914, IT). Le premier monte-charge sur rail se trouve dans la séquence babylonienne d'Intolérance (David Wark Griffith, 1916, USA). Il s'élevait à 35 mètres de hauteur. Le premier film en cinémascope est La Tunique (Henry Coster, 1953, USA). La première utilisation d'un texte intégré à l'image pour remplacer les cartons qui brisaient le rythme du film se trouve dans le Ben-Hur de Fred Niblo (1925, USA). Le musicien Osvaldo Brunetti écrivit, en 1910, la première partition musicale originale pour un film : L'Esclave de Carthage (Luigi Maggi, IT). Restons dans le domaine musical pour signaler que le tournage de La Vie du Christ d'Alice Guy (1906, FR) se déroula au son du gramophone pour sensibiliser les 
acteurs. Terminons pileusement : les faux cils furent inventés pour Intolérance. L'actrice Seena Owen avait ainsi un regard plus profond...

\section{Puis dans le registre : " Le plus grand, la plus grande "...}

On trouve quelques titres de péplums. Le plus grand nombre d'Oscars pour un seul film? Le Ben-Hur de 1959 (William Wyler, USA) : 11. Le plus grand nombre de caméras pour filmer une seule scène? 48 pour la bataille navale du Ben-Hur de 1925. Le plus grand décor? Le Forum romain de La Chute de l'Empire Romain (Anthony Mann, 1963, USA) : 400 mètres sur un terrain de 20 hectares. La plus grande réduction de pellicule au montage? Toujours Ben-Hur (version 1959) : 340000 mètres descendus à 7000. La séquence la plus réduite? Encore Ben-Hur, mais version 1925 : 60000 mètres réduits à 225 mètres pour la course de chars. Finissons par une note vestimentaire. Le plus grand nombre de costumes utilisés? 32000 dans le Quo Vadis? de Mervin LeRoy (1951, USA).

\section{Enfin, le bêtisier cinématographique}

On trouve : un avion qui passe dans Hélène de Troie (Robert Wise, 1955, USA), des fils télégraphiques dans Fabiola (Alessandro Blasetti, 1947, IT/FR) et, au milieu des Perses qui donnent l'assaut de Babylone dans Intolérance, un assistantréalisateur en manches de chemise et cravate ! Le souci maniaque d'exactitude de Cecil Blount DeMille lui fit dépenser 100000 dollars pour envoyer une équipe en Égypte chargée de vérifier la couleur exacte des pyramides. C'était pour Cléopâtre (1934, USA), un film en noir et blanc...

\section{Le mot de la fin}

Gardons pour la fin l'histoire de ce réalisateur, venu du théâtre, qui n'avait jamais été au cinéma et qui croyait que filmer consistait à prendre des photos et à les projeter... Il fallut donc attendre, chaque soir, qu'on lui fasse quitter le plateau sous les prétextes les plus divers pour continuer le film : La Passion (1897, USA), “ filmée » par Henry Vincent. À moins qu'il ne le faille considérer comme le premier film sans réalisateur?

Claude Aziza

Université de la Sorbonne Nouvelle,

Paris III

claude.aziza@laposte.net 